\title{
La prolongation des conflits
}

\section{Rémy Leveau}

\section{(2) OpenEdition}

1 Journals

\section{Édition électronique}

URL : http://journals.openedition.org/conflits/133

DOI : $10.4000 /$ conflits. 133

ISSN : 1777-5345

Éditeur :

CCLS - Centre d'études sur les conflits lilberté et sécurité, L'Harmattan

\section{Édition imprimée}

Date de publication : 21 janvier 1990

ISSN : 1157-996X

\section{Référence électronique}

Rémy Leveau, "La prolongation des conflits », Cultures \& Conflits [En ligne], 01 | hiver 1990, mis en ligne le 07 janvier 2003, consulté le 30 mars 2021. URL : http://journals.openedition.org/conflits/133 ; DOI : https://doi.org/10.4000/conflits. 133

Ce document a été généré automatiquement le 30 mars 2021.

Creative Commons License 


\title{
La prolongation des conflits
}

\author{
Rémy Leveau
}

1 Depuis mars 1988, l'hypothèse d'une tendance à la réduction des conflits influence l'analyse des confrontations " mineures " qui affectent diverses régions du monde. Forgée dans le cadre de la prégnance des rapports Est/Ouest, elle prend de plus en plus mal en compte les évolutions récentes comme le montre le cas afghan où l'évacuation annoncée par les Soviétiques, dès 1988, n'a en rien conduit à la fin du conflit. Dans la logique de gestion des conflits depuis la fin de la Seconde Guerre mondiale, un certain type de rapports de rivalités n'excluait pas en effet la prise en compte d'intérêts communs par des systèmes politiques soucieux d'éviter le pire, et notamment l'engagement dans des situations d'affrontement direct. Dans cette perspective, l'effondrement progressif du communisme et la fin de la tension des deux grands signifiaient à la fois un désengagement soviétique, une pacification des conflits entretenus dans l'intérêt d'une des puissances, une généralisation des sorties de crise, la mise en place d'une ouverture démocratique et une entrée progressive dans l'économie de marché. Certes, l'articulation imaginée entre ces divers facteurs aurait pu constituer un domaine d'interaction créant un nouveau courant positif de relations internationales, mais ces relations tendent à échapper au contrôle des États et à constituer une sorte de domaine transnational où les sociétés civiles gèrent de façon informelle des processus d'évolution donnant parfois l'impression d'une certaine anarchie. De plus l'affaiblissement des tendances lourdes de bipolarisation idéologique crée une sorte de déconnexion des rapports Nord-Sud Qui apparaissent comme secondaires par rapport à un centrage préférentiel des relations des pays du nord de l'Europe au profit de l'Est. Cet affaiblissement des contraintes internationales s'accompagne, en outre, dans les pays du Sud d'un discours sur la démocratie qui se limite le plus souvent à un discours idéologique de demande d'aide et de complicité, sans aller jusqu'à la participation des populations à la gestion des affaires communes suivant des rapports institutionnalisé.

2 Le poids de la croissance démographique globale, celui de l'augmentation de la population urbaine, l'élévation du niveau d'éducation de la jeunesse créent des besoins économiques, politiques et culturels que les États ne sont pas en mesure de satisfaire. Ils laissent alors la place à certaines formes de récupération par les courants 
idéologiques et religieux nouveaux qui apparaissent comme étant seuls capables d'imposer un système de normes, de valeurs, de restriction de consommation à une société composée de jeunes sans emploi et de paysans dépaysannisés. Or, ces condition sociales sont certainement plus favorables à l'apparition de régimes autoritaires de type militaire sur le modèle de l'Amérique du Sud qu'à un contrôle institutionnalisé des gouvernants. Dans ce contexte, le discours démocratique risque de faire partie d'une sorte de mode destinée à attirer des crédits et des subventions. On constate en même temps, il est vrai, l'introduction de certaines formes d'ouverture économique tendant notamment à l'intégration de secteurs partiels d'économie de marché et d'accès à l'économie mondiale. L'apparition de groupes d'entrepreneurs constitue un début de société civile autonome du pouvoir politique qui devrait également créer les éléments d'un pluralisme favorisant à terme une construction démocratique. Mais les appareils économiques d'État défendent souvent avec beaucoup d'énergie leur domaine d'activité et divers exemples prouvent, notamment au Liban, que les entrepreneurs peuvent aussi participer et être subordonnés au jeu des milices. Par ailleurs, les secteurs économiques nouveaux créent des tensions et des inégalités sociales difficiles à gérer sur le plan politique. L'ouverture économique qui est peut-être, à certains égards, plus réelle que l'ouverture politique ne se traduit pas pour autant par un supplément de démocratie. Ces changements politiques et économiques s'accompagnent également d'une évolution très rapide du champ culture1. La diffusion de l'éducation, l'exposition de plus en plus intense aux images et médias échappant au contrôle des gouvernements créent aussi des formes d'ouverture vers l'extérieur particulièrement sensibles chez les jeunes et dans la classe moyenne. Ces groupes vivent aujourd'hui en portant une attention extrême à la vie politique, aux valeurs et comportements auxquels ils sont exposés avec une grande intensité du fait d'une couverture d'images, d'une écoute

Des radios étrangères, d'un trafic clandestin des vidéo cassettes. Cette création d'un nouveau champ culturel ?e forte intensité aboutit le plus souvent à donner à de larges couches de la population l'envie de se situer ailleurs par rapport à l'État et à la société où ils vivent. Mais, comme cet " ailleurs " est inaccessible faute de possibilité d'émigrer ou d'un capital culturel et technique qui permet de relativiser ces perceptions, les lectures et la compréhension de ces influences culturelles ne se traduisent pas obligatoirement par un désir d'intégration à l'État et aux secteurs modernisés de la société. Au contraire, certains des groupes exposés à ces influences réagissent très vigoureusement par une réaffirmation agressive de leur identité culturelle.

Les scénarios, faits à l'occasion des sorties de crise, sous-estiment donc largement les zones d'ombre qui étaient dissimulées p la bipolarisation des conflits et l'imposition de logiques extérieur contraignantes. Le communisme ou la présence américaine entretenait en effet des contrôles et des limitations pouvant servir compenser des soutiens acquis dans d'autres secteurs. Une certaine logique de contrôle de l'intensité des conflits constituait un schéma commun. La disparition progressive de ces affrontements indirects entre superpuissances entraîne l'affaiblissement de ces contraintes comme le démontrent les divers articles de ce numéro. Ne doit-on pas, à partir de là, orienter la réflexion dans une autre direction imaginer des évolutions fondées sur la perpétuation de situations conflictuelles où les acteurs alimentent leur opposition dans généralisation des tensions? Il est frappant de voir, à partir d'exemples afghans, libanais ou sud-américains, s'établir des bas d'une économie 
délinquante assurant l'entretien des conflits échappant assez largement au contrôle dans les États de forces extérieures.

Dans cette perspective, des scénarios d'évolution catastrophique pouvant comporter malgré tout des éléments de stabilisation pot raient être plus facilement développés et argumentés que des schémas optimistes du type transition autoritaire ou libérale vers démocratie et l'économie de marché. On pourrait en effet imaginer que quelques secteurs économiques comme l'énergie, les matières premières, la sous-traitance de fabrications diverses permettent une certaine forme de participation à l'économie mondiale de pays qui refusent d'intégrer des populations nouvellement urbanisées dans un système de relations leur assurant un niveau de vie et une participation décente à la société de consommation. Si cette évolution avait lieu, on assisterait en effet, dans de larges régions, à une marginalisation de vastes ensembles urbains qui ne seraient contrôlés que par des constructions idéologiques et religieuses ainsi que par le recours régulier ou épisodique à la violence à l'état brut aboutissant à des organisations sociales permettant à un coût minimum certaines formes de communication avec l'extérieur. A partir de là, on pourrait assister en effet à une généralisation de l'économie délinquante fondée sur la drogue, les activités de contrebande à grande échelle et les migrations sauvages. Dans cette perspective, une association de pouvoirs militaire et religieux partageant le contrôle des masses devrait suffire à une gestion minimale de systèmes sociaux marginalisés. Nous sommes loin de la transition vers la démocratie. Dans une telle situation, on pourrait imaginer la perpétuation de guerres ruineuses pour les sociétés mais tout à fait profitables pour une minorité qui assure le contrôle et l'encadrement de ces ensembles. L'analyse de Salim Nasr sur le Liban montre à cet égard la créativité du système des milices. I1 faudrait y ajouter son articulation avec des entrepreneurs qui ont, pendant une bonne partie de la guerre, continué à assurer une activité économique à Beyrouth dans des conditions d'efficacité plus grande que celle des pays voisins. Ainsi, l'absence d'encadrement étatique peut créer les conditions de fonctionnement d'un libéralisme sauvage à l'état pur. On assiste dans le cas du Liban ou de l'Afghanistan à la mise en place d'un processus d'autolimitation de la violence et de collaboration entre les acteurs principaux qui la contrôlent. On pourrait imaginer l'explosion progressive de tels systèmes, or, on assiste plutôt à leur perpétuation et peut-être demain à leur extension à un ensemble de pays où l'État a été affaibli de façon à ne plus pouvoir supporter le coût d'un certain ordre social ni la prise en charge de la protection de la population. On doit notamment s'interroger sur la possibilité d'extension de ces situations détériorées au Maghreb, à l'Afrique de l'Ouest ou aux républiques musulmanes de l'Union soviétique. Par opposition, le cas du Nigeria offre un exemple intéressant et, à certains égards, rassurant. Les tensions religieuses et ethniques existent à l'intérieur du système mais le gouvernement fédéral assure une sorte de régulation régionale souvent instable, s'accommodant d'un degré élevé de désordre institutionnalisé. Il maintient ainsi dans la longue durée le fonctionnement d'un ensemble de vaste dimension opérant un contrôle des conflits s'effectuant dans de meilleures conditions que dans les pays voisins. Ne faut-il pas voir là, dans la création d'ensembles régionaux, l'espoir de voir se constituer de nouvelles identités débouchant sur des noyaux politiques stables? L'installation d'un processus de domination globale de la Syrie au Liban permettra-telle par exemple l'imposition d'un nouvel ordre régional de ce type ou débouchera-telle sur la généralisation de l'économie de gang? Les enjeux économiques mis en évidence par Salim Nasr, l'importance de l'économie de la drogue (évaluée à 1,2 
milliard de dollars), contrôlée à 30 \% par les milices, montrent l'ampleur du problème et laissent penser que le maintien des conflits et des tensions fondés sur des alliances contre nature à l'intérieur et à l'extérieur a de fortes chances de se perpétuer.

6 A partir de là, peut-on cependant espérer voir s'établir au Moyen-Orient, au Maghreb ou en Amérique centrale de nouveaux ensembles régionaux articulés sur le système international gui maintiendraient sous contrôle, sans cependant les limiter, la part d'économie souterraine et d'économie délinquante qui alimentent les systèmes locaux tant que des modes de partage de ressources, de solidarité et de contrôle de la violence ne peuvent être établis? Cette évolution peu satisfaisante sur le plan moral pourrait receler cependant les éléments d'un équilibre stable dans la détérioration et la marginalisation de ses composants. Toutefois, la présence latente dans ces ensembles régionaux en décomposition de risque d'accès aux armes nucléaires dont les effets s'étendent à l'environnement international et aux systèmes restés à l'écart dans leur prospérité peut compromettre cette évolution. Cela devrait ouvrer à terme pour un système de contrôle mondial des conflits locaux qui apparaît encore trop coûteux pour le moment à des super-puissances qui espèrent s'isoler un moment pour savourer la fin de leurs affrontements.

\section{RÉSUMÉS}

Depuis mars 1988, l'hypothèse d'une tendance à la réduction des conflits influence l'analyse des confrontations " mineures " qui affectent diverses régions du monde. Forgée dans le cadre de la prégnance des rapports Est/Ouest, elle prend de plus en plus mal en compte les évolutions récentes comme le montre le cas afghan où l'évacuation annoncée par les Soviétiques, dès 1988, n'a en rien conduit à la fin du conflit.

No summary

INDEX

Mots-clés : sociologie des conflits (polémologie)

\section{AUTEUR}

\section{RÉMY LEVEAU}

Professeur de science politique à l'Institut d'Etudes Politiques de Paris 